\title{
Contracepção reversível de longo prazo em adolescentes: benefícios e barreiras
}

Long-term reversible contraception in adolescents: benefits and barriers

Anticoncepción reversible a largo plazo en adolescentes: beneficios y barreras

Nycolle Cavalcanti Silva de Santana ${ }^{1 *}$, Patricia de Albuquerque Chaves ${ }^{1}$, Larissa Oliveira da Silva ${ }^{1}$, Regina Oliveira Vaz ${ }^{1}$, Hirla Vanessa Soares de Araújo ${ }^{1}$, Vivian Conceição Alves Leite Pereira do Lago'.

\section{RESUMO}

Objetivo: Identificar na literatura científica as evidências sobre os fatores que interferem na baixa escolha de métodos de contracepção reversível de longo prazo (LARCs) em adolescentes e seus benefícios para o público em questão. Métodos: Trata-se de uma revisão integrativa da literatura feita a partir do levantamento de artigos nas bases de dados: Literatura Latino-Americana e do Caribe em Ciências da Saúde (LILACS), Medical Literature Analysis and Retrieval System Online (MEDLINE) e Scientific Electronic Library Online (SCIELO) com os descritores: Long-acting reversible contraception, contraception and adolescents, resultando em 2.242 artigos com seleção final de 9 artigos de acordo com os critérios de inclusão e exclusão.

Resultados: Embora os LARCs tenham se mostrado como uma ótima alternativa para prevenir a gravidez na adolescência por sua baixa taxa de descontinuidade, a falta de informação tanto dos profissionais, quanto dos adolescentes faz com que ele não seja a primeira escolha da população em destaque. Considerações finais: Esta pesquisa permitiu observar os principais fatores que interferem na baixa demanda de LARCs, apesar de seus benefícios. Sugere-se mais estudos sobre esses fatores e implementação de estratégias visando mudar essa realidade.

Palavras-chave: Contracepção reversível de longo prazo, Anticoncepção, Adolescentes.

\begin{abstract}
Objective: To identify evidence in the scientific literature on the factors that affect the low choice of long-term reversible contraception (LARCs) in adolescents and their benefits for the target audience. Methods: This is an integrative literature review based on a survey of articles in the following databases: Latin American and Caribbean Literature on Health Sciences (LILACS), Medical Literature Analysis and Retrieval System Online (MEDLINE) and Scientific Electronic Library Online (SCIELO) with the descriptors: Long-acting reversible contraception, contraception and adolescents, resulting in 2,242 articles with a final selection of 9 articles according to the inclusion and exclusion criteria. Results: Although LARCs have been shown to be a great alternative to prevent teenage pregnancy due to its low rate of discontinuity, the lack of information from both professionals and adolescents makes it not the first choice of the highlighted population. Final considerations: This research allowed us to observe the main factors that interfere with the low demand for LARCs, despite their benefits. Further studies on these factors and implementation of strategies to change this reality are suggested.
\end{abstract}

Key words: Long-acting reversible contraception, Contraception, Adolescents.

${ }^{1}$ Centro Universitário dos Guararapes (UNIFG), Jaboatão dos Guararapes - PE.

*E-mail: nycollecsilva@gmail.com

SUBMETIDO EM: 6/2021

PUBLICADO EM: 7/2021 


\section{RESUMEN}

Objetivo: Identificar evidencia en la literatura científica sobre los factores que afectan la baja elección de anticonceptivos reversibles de largo plazo (LARC) en adolescentes y sus beneficios para el público objetivo. Métodos: Se trata de una revisión bibliográfica integradora basada en una encuesta de artículos en las siguientes bases de datos: Literatura Latinoamericana y del Caribe en Ciencias de la Salud (LILACS), Sistema de Análisis y Recuperación de Literatura Médica en Línea (MEDLINE) y Scientific Electronic Library Online (SCIELO) con los descriptores: Anticoncepción reversible de acción prolongada, anticoncepción y adolescentes, resultando en 2.242 artículos con una selección final de 9 artículos según los criterios de inclusión y exclusión. Resultados: Si bien los LARC han demostrado ser una gran alternativa para prevenir el embarazo adolescente debido a su baja tasa de discontinuidad, la falta de información tanto de profesionales como de adolescentes hace que no sea la primera opción de la población destacada. Consideraciones finales: Esta investigación permitió observar los principales factores que interfieren con la baja demanda de LARC, a pesar de sus beneficios. Se sugieren más estudios sobre estos factores y la implementación de estrategias para cambiar esta realidad.

Palabras clave: Anticoncepción reversible de larga duración, Anticoncepción, Adolescentes.

\section{INTRODUÇÃO}

Desde a criação do SUS na década de 80 até os dias de hoje muitos problemas de saúde pública foram solucionados ou pelo menos controlados. Um dos problemas de saúde pública que chama muita atenção nos tempos atuais é a maternidade na adolescência, principalmente devido aos riscos perinatais. Anemia, préeclâmpsia, hemorragia pós-parto e mortalidade materna têm proporções maiores em gestações de adolescentes e o nascimento prematuro, mortalidade perinatal, baixo peso ao nascer e baixo índice de apgar são problemas frequentes em filhos de mulheres jovens (BOROVAC-PINHEIRO A, et al., 2016; WADO YD, et al., 2019).

Além dos riscos perinatais, a gravidez na adolescência traz ainda impactos socioeconômicos, pois ocorre uma grande incidência de abandono escolar, o que reduz a oportunidade de formação educacional, oportunidade no mercado de trabalho, privação socioeconômica e perpetuação de desigualdade social. Principalmente tendo em vista que a gestação precoce ocorre em sua maioria em jovens com baixas condições financeiras. Esse tipo de gestação ainda afeta diretamente a economia pública, pois cabe ao estado todos os gastos com os cuidados infantis, cuidados com impactos físicos maternos, cuidados a abortos espontâneos e posteriormente o auxílio de benefícios a essa mãe/família que não tem uma boa condição financeira e se encontra muitas vezes em risco de vulnerabilidade social (PARKS C e PEIPERT JF, 2016).

Graças ao avançar da ciência, atualmente existe uma grande variedade de métodos contraceptivos disponíveis no mercado para escolha das usuárias, cada um com seus respectivos possíveis efeitos colaterais, porcentagem de eficácia e tempo de duração. Dentre os métodos contraceptivos disponíveis no mercado hoje, os métodos contraceptivos reversíveis de longa duração (LARCs), são os que possuem maior eficácia na prevenção de gestações indesejadas quando comparados aos métodos de curta duração como pílula oral combinada e camisinha (HANSEN RTB e ARORA KS, 2018).

A OMS afirma que qualquer método contraceptivo é indicado independente da idade da pessoa que faz seu uso, as contra indicações a respeito de contracepção não podem ser baseadas na idade do usuário, mas sim em achados clínico-patológicos, hereditários ou até mesmo efeitos colaterais provocados pelo método escolhido. O principal ponto a ser levado em consideração na escolha de um método contraceptivo seria sua eficácia, segurança e sua facilidade de acesso, entendendo sempre o posicionamento do paciente em relação aos seus fatores pessoais, religiosos, culturais e suas preferências (TODD N e BLACK A, 2020).

Estudos trazem ainda que se utilizados de maneira correta é estimado que a taxa de falha do preservativo masculino e da pílula oral combinada seja entre $9 \%$ e $18 \%$ enquanto para métodos DIU, e implantes a falha 
pode ocorrer entre $0,2 \%$ e $0,8 \%$. Apesar da maior eficácia do uso de LARCs em adolescentes, a maioria deles ainda opta pelo uso de métodos contraceptivos orais, que embora seja um método muito eficaz, depende muito da usuária para manter sua eficácia, uma vez que ela é reduzida quando não tomada diariamente como recomendado, o qual é um problema frequente dentre os jovens (ONYEWUCHI UF, et al., 2020; MAES I, et al., 2018).

Tendo em vista a baixa utilização dos LARCs em adolescentes, apesar de sua alta eficácia para um dos problemas mais frequentes na adolescência, esse estudo se faz necessário para identificar os fatores que influenciam nessa baixa escolha do método, tendo como objetivo identificar na literatura científica o que as evidências apontam sobre principais fatores que interferem na escolha de métodos contraceptivos de longo prazo em adolescentes e seus benefícios.

\section{MÉTODOS}

Trata-se de uma revisão integrativa da literatura, cujo método incluiu análise de pesquisas já realizadas, síntese de conhecimentos e reflexões sobre intervenções futuras. O método utilizado tem grande importância para a área de saúde, pois possibilita o profissional da área a ter conhecimento de diversas informações disponíveis no campo científico em um curto espaço de tempo (SOUSA LMM, et al., 2017).

A metodologia selecionada possibilitou averiguar e compreender de modo abrangente as evidências disponíveis até o presente momento na literatura sobre a temática partindo da seguinte questão norteadora: "O que as evidências científicas apontam sobre principais fatores que interferem na escolha de métodos contraceptivos de longo prazo em adolescentes e seus benefícios?".

Após a realização desse questionamento, seguiu-se às outras etapas para elaboração da pesquisa: formulação dos critérios de inclusão e exclusão, definição das informações a serem aproveitadas das pesquisas escolhidas, avaliação rigorosa dos estudos incluídos na revisão integrativa, interpretação dos achados e síntese do conhecimento adquirido ao final da pesquisa.

Para a seleção dos estudos, a busca foi realizada através de levantamento de artigos científicos acessando as bases de dados: Literatura Latino Americana e do Caribe em Ciências da Saúde (LILACS), Medical Literature Analysis and Retrieval System Online (MEDLINE), e Scientific Eletronic Library Online (SCIELO). Os descritores foram selecionados a partir de uma pesquisa no Health Sciences Descriptors (DeCS/MeSH): Long-acting reversible contraception, contraception e adolescents. Foram utilizados também os operadores booleanos OR e AND, permitindo estratégia de busca, da seguinte forma: Long-acting reversible contraception OR contracepion AND adolescents.

Os descritores foram inseridos nessa ordem de forma replicada em todas as bases de dados utilizadas para busca, visando coletar o maior número de estudos na área de interesse. Os artigos escolhidos seguiram aos seguintes critérios de inclusão: Delimitação do tempo no período de 2016 a 2021, contendo texto completo; Idioma Português ou Inglês e artigos disponíveis em sua totalidade e gratuitamente. As matérias de revistas, livros completos e artigos que não respondessem à pergunta norteadora do estudo.

A leitura seletiva dos artigos foi realizada, inicialmente, com análise do título e resumo. Os artigos duplicados foram registrados apenas uma vez, em seguida as informações da leitura completa dos estudos foram interpretadas, resumidas e organizadas, comparando-se os resultados e finalizando os estudos.

\section{RESULTADOS}

Foi identificado um total de 2.242, dos quais 2 foram excluídos por duplicação, resultando em 2.240 estudos rastreados, onde 2.225 foram excluídos por título e resumo e 6 artigos excluídos por não atenderem a questão norteadora. Ao final da pesquisa, foram selecionados 9 artigos para a revisão de literatura (Figura 1). 
Figura 1 - Fluxograma com estratégia de seleção de artigos.

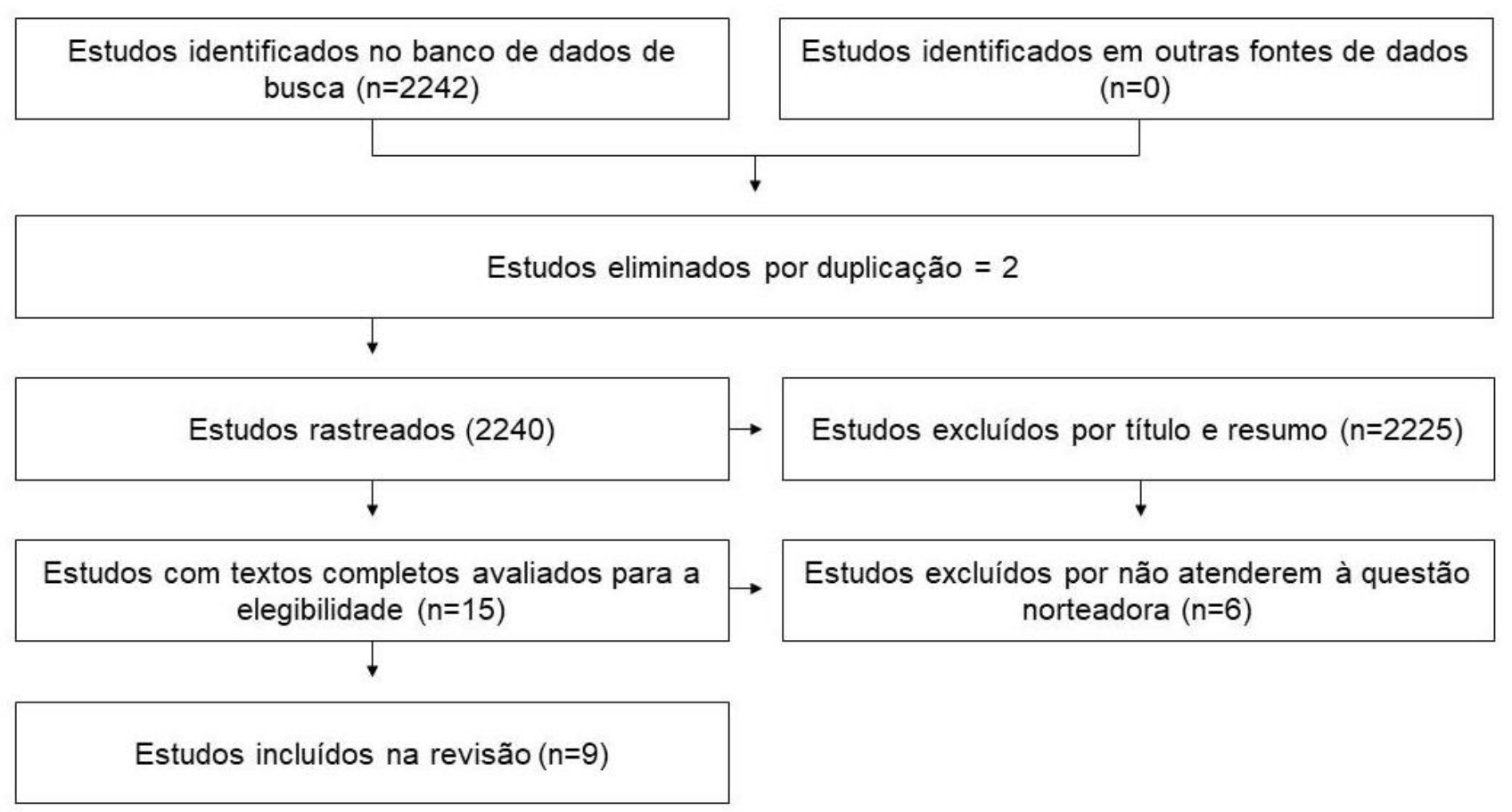

Fonte: Santana NCS, et al., 2021.

Conforme a classificação de análise de pesquisas foram escolhidos 9 artigos originais. O primeiro estudo foi o de Aligne CA, et al. (2020), realizado nos Estados unidos e nos trouxe uma abordagem intervencional clínica, na sequência temos o artigo de Vayngotin T, et al. (2020), também trouxe um estudo intervencional clínico, realizado nos Estados Unidos; Gibbs SE, et al. (2016), realizado nos Estados Unidos, teve como tipo de estudo uma intervenção clínica comparativa; Murphy MK, et al. (2016), realizado nos Estados Unidos através de um estudo clínico qualitativo; Teal SB, et al. (2019), um estudo observacional prospectivo, feito nos Estados Unidos; Hoopes AJ, et al. (2016) apresentou um estudo de coorte observacional, elaborado nos Estados Unidos; Diserens C, et al. (2017) feito na Suíça por meio de um estudo qualitativo; Ezenwaka U, et al. (2020), realizado na Nigéria, teve como tipo de estudo misto qualitativo e quantitativo; e Borovac-Pinheiro A, et al. (2019), no Brasil com um de estudo observacional retrospectivo.

O Quadro 1 mostra de forma objetiva os artigos, apresentando o autor, ano de publicação, foco do estudo e suas conclusões, nos permitindo uma visão ampla das características dos artigos selecionados para análise. 
Quadro 1 - Síntese dos principais achados sobre determinado tema.

\begin{tabular}{|c|c|c|}
\hline Autores (Ano) & $\begin{array}{l}\text { Foco do } \\
\text { estudo }\end{array}$ & Conclusões \\
\hline $\begin{array}{l}\text { ALIGNE CA, } \\
\text { et al. (2020) }\end{array}$ & $\begin{array}{l}\text { Profissionais } \\
\text { que trabalham } \\
\text { com } \\
\text { adolescentes. }\end{array}$ & $\begin{array}{l}\text { Houve um aumento de } 4 \% \text { a } 24 \% \text { no uso de LARCs em adolescentes } \\
\text { que estão cursando o ensino médio e são sexualmente ativas durante o } \\
\text { período de } 2013 \text { a } 2017 \text {, e durante esse mesmo período no restante do } \\
\text { estado de NY a porcentagem de uso aumentou entre } 1,5 \% \text { a } 4,8 \% \text {. }\end{array}$ \\
\hline $\begin{array}{l}\text { VAYNGOTIN } \\
\text { T, et al. (2020) }\end{array}$ & $\begin{array}{l}\text { Adolescentes } \\
\text { que estavam em } \\
\text { um centro de } \\
\text { atendimento de } \\
\text { pronto socorro. }\end{array}$ & $\begin{array}{l}\text { Antes de assistir o vídeo, } 17,7 \% \text { das participantes estavam interessadas } \\
\text { no DIU ou Implante, após assistir o vídeo } 42,3 \% \text { estavam interessados } \\
\text { no DIU e } 35,7 \% \text { interessados no implante, entre as adolescentes que } \\
\text { completaram o estudo } 16 \% \text { iniciaram o LARC após o estudo. Após } \\
\text { assistir o vídeo o interesse entre os adolescentes foi ampliado, porém } \\
\text { não houve uma diferença real na adesão de anticoncepcionais. }\end{array}$ \\
\hline $\begin{array}{l}\text { GIBBS SE, et } \\
\text { al. (2016) }\end{array}$ & $\begin{array}{l}\text { Mulheres com } \\
\text { idade entre } 18- \\
25 .\end{array}$ & $\begin{array}{l}\text { Foram inscritas } 1500 \text { mulheres, delas } 22 \% \text { eram adolescentes entre } 18- \\
19 \text { anos. Entre as adolescentes } 85 \% \text { eram nulíparas, } 77 \% \text { eram brancas } \\
\text { ou hispânicas, } 81,8 \% \text { delas expressaram o não desejo de engravidar } \\
19,5 \% \text { relataram ter múltiplos parceiros nos últimos três meses. As } \\
\text { mulheres na intervenção aumentaram a iniciação, seleção e } \\
\text { aconselhamento do método LARC, tanto as adolescentes quanto as } \\
\text { jovens adultas eram propensas no mesmo nível para o aconselhamento } \\
\text { de LARC, entretanto mulheres nulíparas eram menos propensas a } \\
\text { receber o aconselhamento e a selecionar LARCS em comparação com } \\
\text { mulheres paridas. }\end{array}$ \\
\hline $\begin{array}{l}\text { MURPHY MK, } \\
\text { et al. (2016) }\end{array}$ & $\begin{array}{l}\text { Profissionais de } \\
\text { saúde que } \\
\text { trabalham com } \\
\text { adolescentes. }\end{array}$ & $\begin{array}{l}\text { A falta de treinamento aos profissionais sobre inserção, benefícios e } \\
\text { aconselhamento ao uso de LARCS por adolescentes trazem grandes } \\
\text { impactos sobre a falta de uso desses dispositivos por jovens. }\end{array}$ \\
\hline $\begin{array}{l}\text { TEAL SB, et } \\
\text { al. (2019) }\end{array}$ & $\begin{array}{l}\text { Mulheres com } \\
\text { idade entre } 16 \text { e } \\
45 \text { anos. }\end{array}$ & $\begin{array}{l}\text { As mulheres mais jovens do estudo demonstraram ser mais propensas } \\
\text { a relatar acne nova ou piora, dispareunia, dor pélvica e dismenorreia, } \\
\text { mas o dispositivo se mostrou eficaz para contracepção por até } 5 \text { anos e } \\
\text { tem baixas taxas de descontinuidade. }\end{array}$ \\
\hline $\begin{array}{l}\text { HOOPES AJ, } \\
\text { et al. (2016) }\end{array}$ & $\begin{array}{l}\text { Adolescentes do } \\
\text { sexo feminino } \\
\text { em um centro de } \\
\text { saúde escolar } \\
\text { voltado para a } \\
\text { atenção } \\
\text { primária. }\end{array}$ & $\begin{array}{l}\text { Um maior conhecimento sobre LARCs foi associado à raça branca, } \\
\text { história de relação sexual vaginal, e uso atual ou prévio de LARC. } \\
\text { Também foi possível observar uma maior aceitabilidade de LARC } \\
\text { associado ao histórico de relação sexual vaginal. }\end{array}$ \\
\hline $\begin{array}{c}\text { DISERENS C, } \\
\text { et al. (2017) }\end{array}$ & $\begin{array}{l}\text { Adolescente do } \\
\text { sexo feminino } \\
\text { com idade entre } \\
12 \text { e } 19 \text { anos. }\end{array}$ & $\begin{array}{l}\text { O aconselhamento individual a respeito de métodos contraceptivos } \\
\text { trazem impactos positivos para sua continuidade de uso, assim como a } \\
\text { confidencialidade da consulta. Dos métodos contraceptivos usados, os } \\
\text { LARCs apresentaram uma grande taxa de continuidade no uso. }\end{array}$ \\
\hline $\begin{array}{l}\text { EZENWAKA } \\
\text { U, et al. (2020) }\end{array}$ & $\begin{array}{l}\text { Líderes } \\
\text { comunitários, } \\
\text { formuladores de } \\
\text { políticas, } \\
\text { trabalhadores } \\
\text { da área da } \\
\text { saúde e pais de } \\
\text { adolescentes. } \\
\end{array}$ & $\begin{array}{l}\text { O estudo avaliou diversas barreiras que dificultam o uso de métodos } \\
\text { contraceptivos, entre elas as principais ligadas a fatores } \\
\text { socioeconômicos, falta de informações e educação sexual na grade } \\
\text { curricular escolar, fatores sociais religiosos e familiares, falta de centros } \\
\text { institucionais especializados, bem como a falta de profissionais } \\
\text { capacitados para aconselhamento de métodos contraceptivos. Fica } \\
\text { evidenciado que trabalhar em cima dessas barreiras é fundamental para } \\
\text { estimular o uso de métodos contraceptivos e evitar gravidez indesejada }\end{array}$ \\
\hline $\begin{array}{l}\text { BOROVAC- } \\
\text { PINHEIRO A, } \\
\text { et al. (2019) }\end{array}$ & $\begin{array}{l}\text { Mães } \\
\text { adolescentes } \\
\text { em consultas de } \\
\text { pós-parto e pré- } \\
\text { natal. }\end{array}$ & $\begin{array}{l}\text { Na consulta após o parto o anticoncepcional mais escolhido foram os } \\
\text { IUC (contraceptivos intrauterinos) e implantes subdérmicos. Houve uma } \\
\text { taxa significativa de aumento no uso dos IUC depois da intervenção de } \\
\text { educação no pré-natal. }\end{array}$ \\
\hline
\end{tabular}

Fonte: Santana NCS, et al., 2021. 


\section{DISCUSSÃO}

No estudo de Ezenwaka U, et al. (2020), foram identificados por pais de adolescentes, líderes comunitários, trabalhadores da área da saúde, e formuladores de políticas alguns empecilhos que restringem o acesso do adolescente aos serviços contraceptivos. As principais questões que influenciam na falta de contracepção por adolescentes são: a falta de conhecimento sobre métodos contraceptivos, renda insuficiente para arcar com os custos dos métodos contraceptivos ou até mesmo para arcar com os custos de deslocamento para os centros qualificados para atendimento gratuito de adolescentes, a timidez, normas culturais de gênero e normas culturais religiosas, mistificações a respeito do uso de métodos contraceptivos, atendimento inadequado dos profissionais de saúde, e falta de redes sociais e apoio da comunidade.

Profissionais de saúde são indispensáveis para influenciar e aumentar o uso de métodos LARCs por adolescentes, mas ainda assim muitos profissionais não possuem treinamento para esse tipo de contracepção como vemos no estudo de Murphy MK, et al. (2016) onde dos 16 profissionais entrevistados, quatro eram treinados em inserção do DIU e Implante; cinco profissionais estavam habilitados para inserção dos dois métodos, porém não tinham segurança para realizar o procedimento sem supervisão ou não tinham os equipamentos necessários para disponibilização do método em suas clínicas devido a restrições financeiras; três profissionais nunca haviam sido treinados para inserção de LARCs e quatro haviam apenas sido treinados em um dos métodos.

Alguns estudos demonstram que a taxa de uso de LARCs entre adolescentes aumenta quando os profissionais passam por treinamentos a respeito da escolha do método e aconselhamento. É descrito em Aligne CA, et al. (2020) que o uso de métodos LARCs entre adolescentes subiu consideravelmente mais entre as adolescentes da comunidade em que ocorreu a intervenção com os profissionais de saúde, do que no restante do estado de Nova lorque, onde houve um aumento do uso, mas muito menor.

No estudo de Diserens C, et al. (2017) foi analisado que o contraceptivo mais usado entre adolescentes são as pílulas orais combinadas e uma quantidade muito pequena de mulheres da pesquisa optaram pelo uso de algum LARC. Apesar de um número baixo de mulheres terem escolhido os LARCs, todas as mulheres que os escolheram continuaram fazendo uso do método após 1 ano, enquanto dentre as mulheres que optaram pela pílula houve descontinuidade na utilização durante o mesmo período.

Com orientações sobre os LARCs, sua eficácia e benefício o público jovem tende a fazer mais escolhas de uso desses métodos como é descrito na intervenção de Vayngotin T, et al. (2020). No estudo, entre as jovens que assistiram ao vídeo educacional a respeito de métodos LARCs foi observado um aumento considerável no interesse na utilização do DIU e do implante. Em concordância Borovac-Pinheiro A, et al. (2019) traz que após as orientações feitas em consultas de pré-natal com adolescentes mais da metade das puérperas iniciaram o uso de sistemas intrauterinos ou do implante. Entre as participantes a maioria ainda estudava e já haviam utilizado algum anticoncepcional antes da gravidez. As pílulas contraceptivas chegaram a serem utilizadas por algumas delas, mas dentre as que utilizaram, houve uma porcentagem significante de uso irregular do método.

O esquecimento do uso do método contraceptivo ou falta de uso é algo identificado em muitos estudos com participações de adolescentes, podendo ser justificado pela idade e o nível de escolaridade baixo entre jovens. Portanto este público é mais suscetível à gravidez indesejada e seus impactos. Uma das principais vantagens dos LARCs para adolescentes é o seu método de ação sem a necessidade de atitudes da usuária para que o mesmo possa fazer efeito (VAYNGOTIN T, et al., 2020; BOROVAC-PINHEIRO A, et al., 2019; DISERENS C, et al., 2017).

Em estudo de Gibbs SE, et al. (2016), no que diz respeito ao perfil social das adolescentes, houve predomínio de jovens brancas ou hispânicas. A maioria das mulheres buscou no período um atendimento para planejamento familiar. No entanto, houve percentual considerável quanto à busca de atendimento para a realização do aborto. É notório que o público de mulheres adolescentes faz uma menor busca aos centros de saúde especializados quando comparadas com mulheres mais velhas, e ainda assim grande parte da 
procura é realizada por motivos de complicações na saúde e não para planejamento familiar. Não obstante, fica claro ainda que a maior parte do público que faz a busca dos serviços de saúde é de etnia branca ou hispânica, o que demonstra a vulnerabilidade da população adolescente negra aos riscos de uma gravidez indesejada e seus impactos.

Da mesma forma, em Hoopes AJ, et al. (2016) também foi observado que mais da metade das mulheres do estudo eram de etnia branca/hispânica. Já com relação à situação financeira, a maioria das jovens era de baixa renda. É destacado neste estudo ainda que o acompanhamento de adolescentes através de um programa de saúde e planejamento familiar ligado à escola e desenvolvido de forma gratuita traz impactos positivos no conhecimento dos jovens em relação à LARCs, trazem também um nível maior de escolha e uso desse método por parte deste grupo. Em relação às perguntas que foram realizadas a respeito de como se usa o LARC seu tempo de duração e informações gerais sobre os métodos, mais da metade das participantes responderam corretamente, sendo grande parte delas jovens de etnia branca.

O estudo de Teal SB, et al. (2019), com 1538 participantes, foi realizado para avaliar a eficácia de um método LARC específico, um sistema intrauterino de levonorgestrel. Durante o estudo, foi possível observar um decréscimo na continuidade da utilização do método com o passar dos anos. As interrupções ocorreram em maior parte por eventos adversos, desejo de gravidez, realocação longe de um local de estudo e perda de acompanhamento. Uma porcentagem mínima das pacientes teve uma expulsão parcial ou total do método, sangramento, infecção vaginal. Além disso, algumas mulheres do estudo foram diagnosticadas com cistos ovarianos sintomáticos e optaram por descontinuar o método devido a isso. Em relação à gravidez, foram identificados no total 9 gestações durante os 5 anos de uso e entre essas, 6 gestações foram ectópicas. Portanto fica comprovado que o método LARC além de eficaz, traz riscos baixos quanto à expulsão, problemas clínicos e efeitos colaterais.

Evidenciou-se nesse estudo que a educação em saúde é fundamental para um aumento no interesse e uma maior adesão de adolescentes aos LARCs e consequentemente uma redução nos índices de gravidez na adolescência, pois muitas jovens não conhecem ou possuem ideais errôneas sobre métodos de contracepção reversíveis de longa duração. (ALIGNE CA, et al., 2020; EZENWAKA U, et al., 2020; HOOPES AJ et al., 2016; VAYNGOTIN T, et al., 2020).

Outros fatores como timidez, déficit de comunicação familiar sobre saúde sexual, medo dos efeitos colaterais e de deslocamento do dispositivo, mau acolhimento, falta de privacidade durante a consulta, crenças religiosas, normas culturais de gênero e falta de dinheiro para arcar com os custos do método ou com transporte para se deslocar até algum local que o faça gratuitamente. Ao observar essa dificuldade no acesso aos LARCs por parte dos adolescentes, nota-se a importância de uma facilitação no acesso a esse serviço (EZENWAKA U, et al., 2020; TEAL SB et al., 2019; MURPHY MK et al., 2016).

\section{CONSIDERAÇÕES FINAIS}

Os maiores benefícios do uso de LARCs em adolescentes é a baixa taxa de descontinuidade do método e o fato de não depender da usuária para ser eficaz, como no caso dos métodos de contracepção de curta duração, onde alguns exigem um esforço diário que muitas adolescentes não conseguem manter, o que acaba resultando em uma gestação não planejada. Quanto aos fatores que interferem na escolha dos adolescentes com relação aos LARCs, pode-se destacar: a desinformação do paciente e do profissional de saúde e a dificuldade no acesso gratuito desse serviço ocasionada pela falta de renda própria para arcar com os custos do deslocamento e pela falta de dialogo com adequado com os pais sobre sexualidade. Dessa maneira, sugere-se mais estudos sobre esses fatores e implementação de estratégias visando mudar essa realidade.

\section{REFERÊNCIAS}

1. ALIGNE CA, et al. Impact of the Rochester LARC Initiative on adolescents' utilization of long-acting reversible contraception. American Journal Of Obstetrics And Gynecology, 2020; 222(4S): S890.e1-S890.e6. 
2. BOROVAC-PINHEIRO A, et al. Adolescent Contraception Before and After Pregnancy-Choices and Challenges for the Future. Revista Brasileira de Ginecologia e Obstetrícia, 2016; 38(11): 545-551

3. BOROVAC-PINHEIRO A, et al. Empowering Adolescent Mothers in the Choice of Contraceptive Methods at the Postpartum Period: Avoiding a Subsequent Pregnancy. Revista Brasileira de Ginecologia e Obstetrícia, 2019; 41(10): 607-612.

4. DISERENS C, et al. Adolescents' contraception continuation in Switzerland: a prospective observational study. Swiss Medical Weekly, 2017; 147(3940): 39-40.

5. EZENWAKA U, et al. Exploring factors constraining utilization of contraceptive services among adolescents in Southeast Nigeria: an application of the socio-ecological model. BMC Public Health, 2020; 20(1):1162.

6. GIBBS SE, et al. Long-Acting Reversible Contraception Counseling and Use for Older Adolescents and Nulliparous Women. The Journal of adolescent health: official publication of the Society for Adolescent Medicine, 2016; 59(6):703709

7. HANSEN RTB; ARORA KS. Consenting to invasive contraceptives: an ethical analysis of adolescent decision-making authority for long-acting reversible contraception. Journal Of Medical Ethics, 2018; 44(9): 585-588

8. HOOPES AJ, et al. Knowledge and Acceptability of Long-Acting Reversible Contraception Among Adolescent Women Receiving School-Based Primary Care Services. Journal Of Primary Care \& Community Health, 2016; 7(3): 165-170.

9. MAES I et al. Knowledge, attitudes and practices of East Flemish general practitioners towards subscribing LARCs for adolescents. Facts, views \& vision in ObGyn, 2018; 10(1): 39-44.

10. MURPHY MK, et al. Interdependent Barriers to Providing Adolescents with Long-Acting Reversible Contraception: Qualitative Insights from Providers. J Pediatr Adolesc Gynecol, 2016; 29(5): 436-442.

11. ONYEWUCHI UF, et al. Improving LARC Access for Urban Adolescents and Young Adults in the Pediatric Primary Care Setting. Clinical pediatrics, 2019; 58(1): 24-33.

12. PARKS C, PEIPERT JF. Eliminating health disparities in unintended pregnancy with long-acting reversible contraception (LARC). American journal of obstetrics and gynecology. 2016; 214(6): 681-688.

13. SOUSA LMM, et al. A metodologia de revisão integrativa da literatura em enfermagem. Revista investigação em enfermagem, 2017; 21(2): 17-26.

14. TEAL SB et al. Five-Year Contraceptive Efficacy and Safety of a Levonorgestrel 52-mg Intrauterine System. Obstetrics \& Gynecology, 2019; 133(1): 63-70.

15. TODD N, BLACK A. Contraception for Adolescents. Journal of clinical research in pediatric endocrinology, 2020; 12:28-40.

16. VAYNGORTIN T, et al. Adolescents' Acceptance of Long-Acting Reversible Contraception After an Educational Intervention in the Emergency Department: A Randomized Controlled Trial. The western journal of emergency medicine, 2020; 21(3): 640-646.

17. WADO YD et al. Pregnancy and early motherhood among adolescents in five East African countries: a multi-level analysis of risk and protective factors. BMC Pregnancy Childbirth, 2019; 19(1): 59. 